
Aug -08-00 $16: 38$

From-NIST-APTD

$+3018693536$

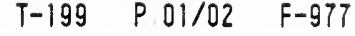

NaT

FAX SHEET $303497 \quad 5204$

NATIONAL INSTITUTE OF STANDARDS AND 'TECLNOL()(i)

Gaillersburg, MD

FAX Number (301) 869.3536

TO

Gerry Blessing

$f_{a x}: 301-926-9524$

IIRỌM Kevin Junes $\times 5486$

As discussed. Do you see a fit with ultrasonics efforts? Clayton suggested characterization of materials properties (for opto-electronie materials) of lime-focels thansducon. Further thoughts? Thanks,

Date 8-8-00

-Kevin

Number of Pages: This one plus _ 2 - unfunded activity

Confirmation Number (301) 975-6600

- applications, have 


\section{Clayton Teague}

From: Gordon W. Day [gwday@boulder.nist.gov]

Sent: Thursday, August 03, 2000 5:30 PM

To: NIST Division Chiefs, OOP Advisory Board

Subject: New Edition, Optoelectronics at NIST

Division Chiefs,

Many of you will recall that, in 1996, NIST published a document called "Optoelectronics at NIST," NISTIR 5054, which contained one page descriptions of about 50 "projects" in optoelectronics|from throughout the NIST Laboratories. It was distributed widely, both within NIST and outside, but is now dated and out of print. Informal feedback suggests that it was useful in fostering collaboration within NIST, and making external contacts.

The Office of Optoelectronics Programs has been asked to develop a new version of the document, organized in such a way as to emphasize NIST efforts as a whole and de-emphasize internal organizational distinctions. We want to portray "one face for NIST in optoelectronics" to the rest of the world, while permitting the reader to identify the individuals and organizations within NIST that are working in areas of interest. To that end, the Office Advisory Board has updated the format for the new edition. The project sheets will be similar to those in the last edition, but they will be grouped by subject area and there will be a key-word index.

If you would like to have your projects in optoelectronics included in the next edition, could you please

1. Review the table of contents of NISTIR 5054 for projects from your Division. If you don't have a copy of the document handy, a Word file of the ToC is attached. If you would like to have the full text of any of the descriptions, send us an email at optoelectronics@boulder.nist.gov with your fax number and we will provide what you need.

2. Identify projects from your Division that you would like to have included in the new version and arrange to have new or updated project sheets prepared. Descriptions should be prepared in the format of the attached model: "High speed optoelectronic measurements". Details of the format are contained in the notes to the model description. [There are now many more collaborative, cros"s Division/Laboratory, projects than there were in 1996. These should be reported only once, but with multipte-contacts as appropriate.]

3. Send the new sheets electronically (Word format preferred) to optoelectronics@boulder.nist.gov by Sept. 1. I realize that this is a busy time of the year, but experience suggests that it takes about an hour to prepare a project sheet. I hope that your project leaders will recognize the benefits and pot find it too burdensome.

Many thanks for your assistance in this project.

Gordon Day

cc: OOP Advisory Board

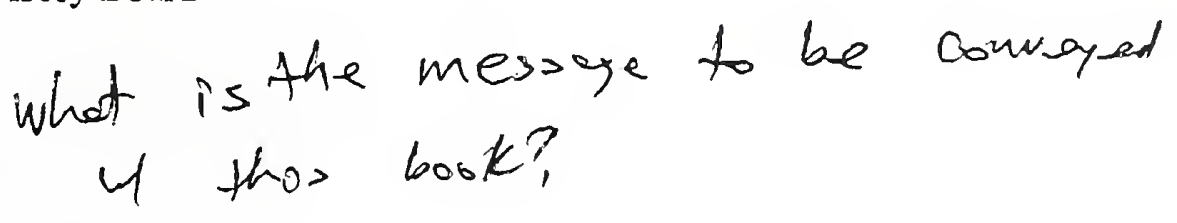


$\curvearrowleft \curvearrowright$ 


\section{OPTOELECTRONICS AT NIST}

Optoelectronics Division

Electronics and Electrical Engineering Laboratory

National Institute of Standards and Technology

Boulder, Colorado 80303-3328

September 1996

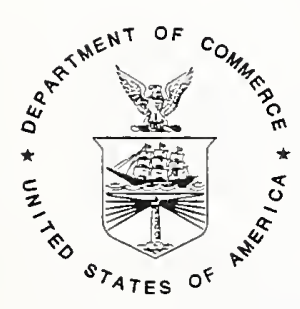

U.S. DEPARTMENT OF COMMERCE, Michael Kantor, Secretary TECHNOLOGY ADMINISTRATION, Mary L. Good, Under Secretary for Technology NATIONAL INSTITUTE OF STANDARDS AND TECHNOLOGY, Arati Prabhakar, Director 



\section{TABLE OF CONTENTS}

Preface vii

Electronics and Electrical Engineering Laboratory

Electricity Division, 811.00

Electronic Instrumentation and Metrology Group, 811.02

Short-Duration Electrical Pulse Generators . . . . . . . . . . . . . . 1

Electro-Optic-Based Electrical Waveform Measurements ............. 2

Electrical Systems Group, 811.05

Electro-Optical Measurement of Surface Charge ................ 3

Optical Current Transducers for Electric Utility Applications . . . . . . . . . 4

Electronic Information Technologies Group, 811.06

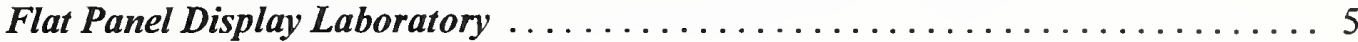

Semiconductor Electronics Division, 812.00

Materials Technology Group, 812.01

Optical Characterization Project ........................ 6

Nanometrology for Compound Semiconductor Materials and Processes . . . . . . 7

Electromagnetic Fields Division, 813.00

Fields and Interference Metrology Group, 813.07

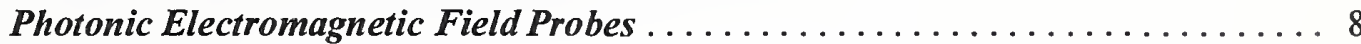

Electromagnetic Technology Division, 814.00

Magnetic Instruments and Materials Characterization .............. 9

Optoelectronics Division, 815.00

Sources and Detectors Group, 815.01

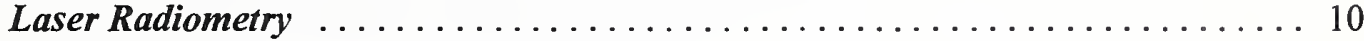

High-Speed Measurements . . . . . . . . . . . . . . . . . . 11

Fiber and Integrated Optics Group, 815.02

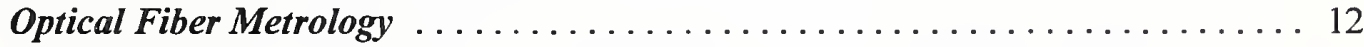

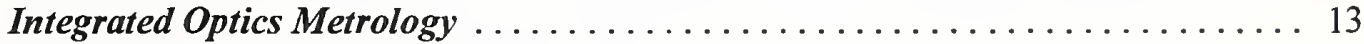

Optical Components Group, 815.03

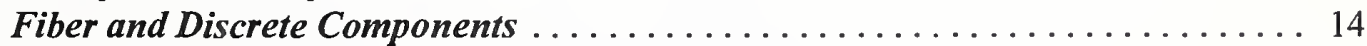

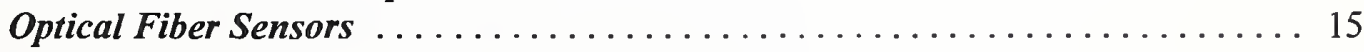

Optoelectronic Manufacturing Group, 815.04

Semiconductor Materials and Devices . . . . . . . . . . . . . . . 16

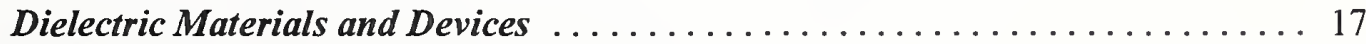


Manufacturing Engineering Laboratory

Precision Engineering Division, 821.00

Mid-Scale and Complex Form Metrology Group, 821.12

Standard Reference Materials for Fiber and Ferrule Geometry .......... 18

Mid-Scale and Complex Form Metrology, 821.12 and Nano-Scale Metrology Group, 821.14

Diode Lasers for Length Measurement . . . . . . . . . . . . . . . . . . . 19

Chemical Science and Technology Laboratory

Biotechnology Division, 831.00

Biomolecular Materials Group, 831.04

Bacteriorhodopsin for Information Storage and Retrieval

Surface and Microanalysis Science Division, 837.00

Surface Dynamical Processes Group, 837.03

Nonlinear Optical Diagnostics of Interfaces and Thin Films (see page 38)

Electronic Structure and Surface Characterization of Oxides . . . . . . . . . . 21

Vibrationally-Resolved Near-Field-Scanning Optical Microscopy .......... 22

Analytical Chemistry Division, 839.00

Spectrochemical Methods Group, 839.01

Fiber and Integrated Optic Chemical Sensors ................... 23

Physics Laboratory

Electron and Optical Physics Division, 841.00

Photon Physics Group, 841.01

Extreme Ultraviolet (EUV) Optics Development and Characterization . . . . . . 24

Far Ultraviolet Physics Group, 841.02

Vacuum Ultraviolet (VUV) Detector Radiometry ................. 25

Electron Physics Group, 841.03

Magnetic Characterization by Confocal MOKE Imaging . . . . . . . . . . . . 26

Characterization of Materials by Optical Emission in Scanning Tunneling

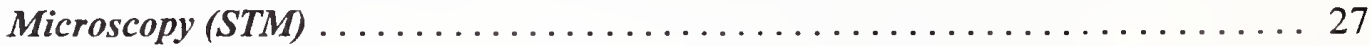

Nanofabrication with Atom Optics . . . . . . . . . . . . . 28

Atomic Physics Division, 842.00

Atomic Spectroscopy Group, 842.01

Atomic Data for High Intensity Lighting . . . . . . . . . . . . . . 29

Plasma Radiation Group, 842.03

Nanoscale Surface Modification for Optical Components . . . . . . . . . . . . 30

Laser Cooling and Trapped Atoms Group, 842.04

Atomic Photonics 
Optical Technology Division, 844.00

Optical Temperature and Source Group, 844.03

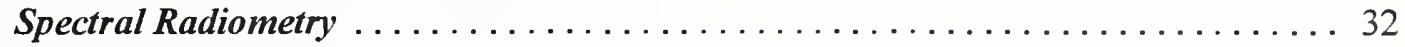

Optical Properties and Infrared Technology Group, 844.04

Infrared Metrology . . . . . . . . . . . . . 33

Optical Sensor Group, 844.05

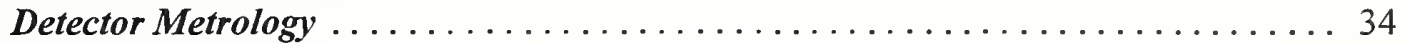

Laser Applications Group, 844.06

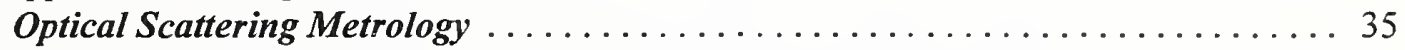

Near-Field Optics and Microscopy for Nanoscale Optical Characterization . . . . . 36

Applications of Femtosecond Terahertz Spectroscopy . . . . . . . . . . . . 37

Nonlinear Optical Diagnostics of Interfaces and Thin Films . . . . . . . . . 38

Time and Frequency Division, 847.00

Optical Frequency Measurements Group, 847.80

Optical Frequency Measurements . . . . . . . . . . . . . . . . . 39

Quantum Physics Division, 848.00

Ultrashort Laser Pulse Interactions with Materials . . . . . . . . . . . . 40

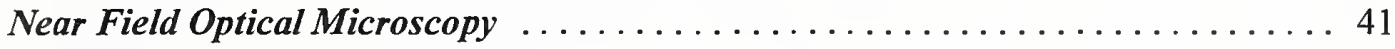

Optical Coherent Control ........................... 42

Materials Science and Engineering Laboratory

Ceramics Division, 852.00

Division Scientists, 852.00

Optoelectronic Crystals: Linear and Nonlinear Optical Interactions . . . . . . . 43

Optical Materials Group, 852.05

Wide-Bandgap Semiconductors for Short-Wavelength Photonic Devices . . . . . . 44

Ferroelectric Oxide Thin Films for Photonics .................... 45

Polymers Division, 854.00

Electronics Applications Group, 854.11

Processing and Lifetime of Light Emitting Polymers . . . . . . . . . . . 46

Metallurgy Division, 855.00

Magnetic Materials Group, 855.11

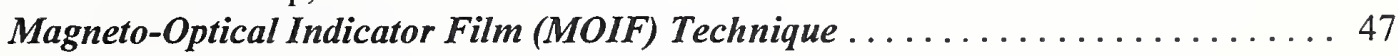

Computer Systems Laboratory

Advanced Systems Division, 875.00

Display and Storage Group, 875.18

Hybrid-Optical Pattern Recognition and Neural Networks . . . . . . . . . 48

Volume Holographic Storage ........................ 49 


\section{Preface}

Optoelectronics comprises the technologies for generating, amplifying, detecting, guiding, and modulating optical radiation. These are broad enabling technologies with applications as diverse as communications, printing, display, data storage, medicine, and sensing. NIST's involvement in optoelectronics is correspondingly diverse and scattered through eighteen divisions in six of the NIST Laboratories. Much of it represents traditional NIST efforts to provide the optoelectronics industry with measurement technology and standards. Some of it involves the use of optoelectronics to achieve NIST's goals in other technologies or in basic research. All of it represents expertise and resources that may be applicable to problems well removed from those on which they are currently focused.

This document is a product of efforts by the ad hoc NIST Optoelectronics Committee to encourage more coordination and collaboration among NIST researchers in the field and more efficient use of NIST resources. Frequently, similar work is being undertaken in different parts of NIST, for very different purposes or applications. The primary audience is NIST scientists, engineers, and managers, but the document is also being made available externally to enable others in the optoelectronics community to identify work at NIST that could be of interest to them.

Forty-nine projects are described. The smallest involves less than one full time equivalent (FTE) staff member, the largest, eighteen. Altogether, approximately 170 FTE staff work in the field. Each project description was prepared by the project leader and contains information about the size of the project, its objectives, constituency, and major current tasks. Additional background information provides some perspective.

Financial support for work reported here comes from many sources. A large portion of the work is supported by appropriated NIST funds. For purposes of this document, various types of NIST funding are not distinguished. Another significant portion is contract research performed for other government agencies. Other sources of funding are too numerous to classify, but include support received through Cooperative Research and Development Agreements (CRADAs) and calibration fees.

Information on staff and staff type should not be regarded as exact, but is included to provide an indication of the size of each project. As defined here, professional staff include NIST employees with degrees in a technical field. Contractors are generally professionals in the field who are under contract to NIST, either directly or through another organization. Guest researchers are professionals from other organizations, normally supported entirely or primarily by their home organization. Technicians listed here are NIST employees. All of these categories may include students.

An electronic version of this document is available at http://www.boulder.nist.gov/div815/oenist96.htm. It is expected that both printed and electronic versions will be revised annually. Corrections, comments, and suggestions are welcome and should be sent by email to optoelectronics@boulder.nist.gov. The work of Annie Smith in preparing the written version and Robert $\mathrm{L}$. Peterson in preparing the electronic version is greatly appreciated.

Gordon W. Day, Optoelectronics Division, EEEL, gwday@boulder.nist gov

Theodore D. Doiron, Precision Engineering Division, MEL, theodore.doiron@nist.gov

Stephen W. Freiman, Ceramics Division, MSEL, stephen.freiman@nist.gov

Debra L. Kaiser, Ceramics Division, MSEL, debra.kaiser@nist.gov

Eung-Gi Paek, Advanced Systems Division, CSL, paek@nist.gov

Albert C. Parr, Optical Technology Division, PL, albert.par@@nist.gov

Steven W. Robey, Surface and Microanalysis Science Division, CSTL, steven.robey@nist.gov

Michael A. Schen, Polymers Division, MSEL, michael.schen@nist.gov

David. G. Seiler, Semiconductor Electronics Division,EEEL, david seiler@nist.gov 



\section{Short-Duration Electrical Pulse Generators}

\section{Project Leader/Contact:}

N. Paulter, (301) 975-2405, paulter@eeel.nist.gov

\section{Approximate staff (FTE):}

0.3 Professional, 0.1 Technician

Funding sources:

NIST (80\%), Other Agency (20\%)

\section{Objective:}

Develop well-characterized short-duration electrical pulses for determination of the impulse response of the oscilloscope used in Special Test. This effort supports the high-speed oscilloscope and pulse generator industry.

\section{Constituency:}

This effort primarily supports the high-speed oscilloscope and pulse generator instrumentation market by providing calibration support for the Electricity Division's pulse parameter special test services requested by U.S. industry and government laboratories. In addition, some companies request traceability to NIST for their high-speed oscilloscopes (bandwidth exceeding $20 \mathrm{GHz}$ ).

\section{Major current tasks:}

- Laser-based measurement system development

- Photoconductor device development

- Device package development

- Device/package electrical characterization

\section{Additional information:}

The project was started in Boulder about 1990 with the purchase of the YAG system (consisting of YAG, IR pulse compressor, frequency doubler, visible pulse compressor). Additional equipment purchases necessary to implement the laser-based measurement system were not made until the work was moved to Gaithersburg (about 1992), after which time it was determined that the YAG system was not capable of providing a stable (over hours) optical pulse train. Funding did not permit purchase of a suitable replacement laser system until inexpensive short-pulse diode-laser-based systems became available. GaAs photoconductors were developed using low-temperature-grown GaAs epilayers that provide scopelimited ( $\sim 18 \mathrm{ps}$ ) measurements of the photoconductor response to $5 \mathrm{ps}$ optical pulses (from YAG). The photoconductor package was developed to minimize the effects of impedance discontinuities between the scope and package connections and of reflections from the backward-propagating pulse. Target electrical pulse parameters are 0.25 to $0.5 \mathrm{~V}$ variable peak amplitude into $50 \Omega$, pulsewidth between 0.5 and 1.5 ps; flat baseline over a $10 \mathrm{~ns}$ epoch; and spurious signals less than $1 \%$ of peak amplitude (none about 100 ps after peak). 


\section{Electro-Optic-Based Electrical Waveform Measurements}

Project Leader/Contact:

N. Paulter, (301) 975-2405, paulter@eeel.nist.gov

Approximate staff (FTE):

0.3 Professional, 0.1 Technician

Funding sources:

NIST (50\%), Other Agency (50\%)

Objective:

Develop electro-optic-(eo)-based techniques for measuring electrical waveform parameters, such as rms voltage, and for profiling high-voltage pulses.

\section{Constituency:}

This is a new project area; consequently, clientele has not been firmly established. Interest in using eo-based rms and power measurement techniques have been expressed by the U.S. Army and U.S.

Navy, and work was funded by ATP for profiling high-voltage electrical pulses.

\section{Major current tasks:}

- Broadband rms voltage measurement system

- High-voltage pulse profiling

Additional information:

A prototype rms voltage measurement system was developed providing about $\pm 1 \%$ uncertainty up to 1 $\mathrm{MHz}$. Work in this area has stopped due to lack of sponsor(s) to cost share further improvements to the system, which has since been disassembled. A high-voltage pulse measurement system was assembled but funds (ATP) were discontinued due to cancellation of the external program. 


\section{Electro-Optical Measurement of Surface Charge}

Project Leader/Contact:

Ken Stricklett, (301) 975-3955, ken.stricklett@nist.gov

Approximate staff (FTE):

0.25 Professional

Funding sources:

NIST (100\%)

Objective:

Develop techniques for measuring surface charge in a RF plasma environment and on dielectric materials in general.

\section{Constituency:}

Semiconductor industry

Major current tasks:

- Conduct a comprehensive literature search

- Design, construct, and characterize a prototype electro-optical charge measuring device.

\section{Additional information:}

The project evaluates electro-optical techniques such as those using the Pockels effect for the measurement of the temporal behavior of surface charge distributions. The proposed measurement system may be adapted to provide in situ two-dimensional surface charge distributions in real time. This facility impacts ongoing research at NIST in plasma processing of semiconductors by enabling measurement of the effect of residual charge on semiconductor surfaces. 


\section{Optical Current Transducers for Electric Utility Applications}

\section{Project Leader/Contact:}

Gerald J. FitzPatrick, (301) 975-2737, fitzpa@eeel.nist.gov

Approximate staff (FTE):

0.3 Professional

Funding sources:

NIST (100\%)

\section{Objective:}

Develop methods for the calibration of optical current transducers (OCTs) designed for use in electric power metering over the range from 100 to $10,000 \mathrm{~A}$ with uncertainties of $0.1 \%$ or less, and protective relaying applications with uncertainties of less than $5 \%$.

\section{Constituency:}

Electric utilities are interested in this work since traceability to NIST is common, and sometimes required by law. OCT manufacturers are interested since a NIST calibration defines one aspect of the performance of their OCTs.

\section{Major current tasks:}

- Calibration of OCTs using analog techniques

\section{Additional information:}

The project on OCT evaluation and testing techniques began in 1991 in response to interest expressed by manufacturers and electric utilities. OCTs were considered prime candidates as replacements for oilfilled current transformers (CTs), particularly by the Tennessee Valley Authority (TVA) and Bonneville Power Administration (BPA), after a number of them failed catastrophically. At first, an OCT was built at NIST for developing testing techniques. Additional funding was supplied by the TVA, which had a number of test OCTs installed in their transmission system. A CRADA with a major equipment supplier was created to obtain a commercial OCT for testing at NIST. Curve-fitting was successfully applied to measured digitized waveforms for comparison of the output of OCTs with reference CTs. OCT scale factors (current ratio or transconductance) and phase errors were determined with $0.1 \%$ uncertainties. Analog comparator techniques are presently being developed to achieve the same uncertainties. 


\section{Flat Panel Display Laboratory}

\section{Project Leader/Contact:}

Bruce F. Field, (301)975-4230, field@eeel.nist.gov

Edward F. Kelley, (301) 975-3842, kelley@eeel.nist.gov

\section{Approximate staff:}

3 Professionals

\section{Funding sources:}

NIST $(100 \%)$

\section{Objective:}

Develop the measurement support needed by domestic industry and users to assess the quality of electronically displayed information.

\section{Constituency:}

Manufacturers (U.S.) who use, or will be using, flat panel displays in their products, e.g., notebook and desktop computer manufacturers; standards committees that rely on NIST technical expertise for the development of flat panel display standards; display test equipment manufacturers and users who implement NIST-developed test procedures.

\section{Major current tasks:}

- Display quality measurements and metrics

- Flat panel display measurement standards

- Diagnostics for display measurement methods

- Small-area contrast measurements

- Display standards metrological evaluations

\section{Additional information:}

NIST research on electronic display measurement issues began in 1992 with the creation of the Flat Panel Display (FPD) Laboratory to address the display-quality metrology needs of the emerging flat panel display industry and its associated users. Many display-quality measurement issues need to be identified and resolved to provide a solid measurement basis to evaluate and specify

application-dependent displays. The staff works closely with domestic and international standards organizations to develop a meaningful suite of display measurements to characterize display performance and provide a uniform language for product comparison and specification. The FPD Laboratory has been called upon to evaluate measurement procedures in existing standards and participate in interlaboratory comparisons concerning display measurement issues. The staff is involved in developing unambiguous display measurement procedures that can be accurately replicated in any display metrology laboratory and provide reproducible results in interlaboratory comparisons. Collaborations are in progress with display measurement equipment manufacturers and FPD manufacturers to refine display measurement methods and evaluation criteria. Development of diagnostics for display measurements, and development of associated SRMs, are also underway. 


\section{Optical Characterization Project}

\section{Project Leader/Contact:}

Paul Amirtharaj, (301) 975-5974, paul@apollo.eeel.nist.gov

Approximate staff (FTE):

3.5 Professionals, 2 Guest Researchers

(approximately $50 \%$ of the staff time is devoted to optoelectronics)

Funding sources:

NIST (80\%), Other Agency (10\%), Other (10\%)

\section{Objective:}

Develop robust optical probes for analyses of technologically important semiconductor materials and heterostructures.

\section{Constituency:}

Semiconductor metrology equipment manufacturers, wafer qualification persons

\section{Major current tasks:}

- Use perturbation techniques for resonant excitation spectroscopy system to investigate defects and strain effects in compound semiconductors.

- Develop spectroscopic measurements and two-dimensional scanning capabilities for alloycomposition/defect-distribution mapping for wafer qualification, and apply to epitaxial AlGaAs.

- Maintain optical facilities, including Raman, infrared absorption, photoluminescence, photoreflectance, and spectroscopic ellipsometry.

\section{Additional information:}

Manufacturers of electronic components for a wide variety of applications extending from digital circuitry for computers to light emitters for optical communication need reliable analytical methods and well-established standards for characterizing the behavior of elemental and compound semiconductor materials. Optical and electrical activity form the foundation of all the major electronic devices in the $\mathrm{RF}$, microwave, microelectronic, and optoelectronics industries. Optical probes for materials characterization are attractive and powerful for a variety of reasons. They are contactless and nondestructive, compatible with any transparent ambient, capable of remote sensing, and compatible with hostile environments. Hence they are useful for in situ probing during growth and processing. "Table-top" procedures can be easily implemented by manufacturers. The continual reduction in feature size for increased packing density and response time for high-speed performance places stringent demands on the current analytical probes. 


\section{Nanometrology for Compound Semiconductor Materials and Processes}

\section{Project Leader/Contact:}

Joseph Pellegrino, (301) 975-2123, joe@apollo.eeel.nist.gov

\section{Approximate staff (FTE):}

2 Professionals, 2 Technicians, 1 Guest Researcher

(approximately $50 \%$ of the staff time is devoted to optoelectronics)

Funding sources: NIST (82\%), Other Agency (8\%), Other (10\%)

\section{Objective:}

Develop advanced measurement methods and Standard Reference Materials for III-V based materials growth and characterization technologies. Support industry standardization of measurement practices and other optoelectronics research at NIST by providing specialized samples.

Constituency: Epi-layer growth manufacturers, optoelectronics device manufacturers

\section{Major current tasks:}

- Develop x-ray-based in situ metrology for real-time composition and growth-rate probes.

- Establish optical probes for in situ metrology to determine thickness, composition, and roughness during growth.

- Implement focused ion beam lithography to define specialized compound semiconductor devices.

- Determine nonlinear properties of electronic/optoelectronics materials for high speed optical switching.

- Characterize the optical, transport, and structural properties of III-V semiconductor heterointerfaces for optimization of device performance.

\section{Additional information:}

The nanoelectronics project provides technological leadership to semiconductor manufacturers by developing and evaluating the methods, tools, and artifacts needed to advance semiconductor nanometrology for the RF, microwave, microelectronics, and optoelectronics industries. It does this by providing compound-semiconductor device manufacturers with advanced metrological techniques and models to improve device manufacturability, performance, and reliability. The project includes growth and analysis of III-V epitaxial layers in order to help industries more reliably produce nanostructure devices. Plans include the development, implementation, and evaluation of both x-ray and optical in situ MBE probes (x-ray fluorescence to determine composition and thickness during growth, in situ ellipsometry of MBE layers for determining thickness, composition, and roughness); x-ray diffraction techniques for determining interface properties of superlattices and heterostructures; focused-ion-beam lithography for patterning low-dimensional test structures and small $(\leq 0.1 \mu \mathrm{m})$ device structures such as quantum lines and dots. The in situ metrology effort has impact on the optoelectronics industry where materials require careful tailoring of composition and dopants. 


\section{Photonic Electromagnetic Field Probes}

Project Leader/Contact:

Keith D. Masterson, (303) 497-3756, masterso@boulder.nist.gov

Approximate staff (FTE):

1 Professional, 0.5 Student

Funding sources:

NIST (85\%), Other Agency (12\%), Other (3\%)

\section{Objective:}

Develop advanced measurement methods and standard field probes for electromagnetic fields and support industry's standardization activities.

\section{Constituency:}

NIST, DoD, DoE, NASA, commercial test laboratories, industry

\section{Major current tasks:}

- Standard rf dipole

- Miniature rf dipole for high spatial resolution of EM-fields

- Vertical cavity semiconductor laser based probe

- Concentric loop antenna system (CLAS) for emissions testing

\section{Additional information:}

NIST research on EM-field probes with photonic links began in 1980. The research has focused on broadband, high frequency probes with passive electrooptic modulators and resistively tapered dipole antennas that are suitable for critical microwave and time domain applications. Systems have been fabricated for DoD and DoE. Electric field sensitivity on the order of $10^{-2} \mathrm{~V} \cdot \mathrm{m}^{-1} \cdot \mathrm{Hz}^{-1 / 2}$ has been obtained. Ongoing research is directed toward increasing the sensitivity, bandwidth, and environmental stability for these probes. The CLAS utilizes loop antennas with diametrically opposed, impedance loaded gaps that simultaneously measure components of the $\mathrm{E}$ and $\mathrm{H}$ fields. It is suitable for near-field measurements. The system has been adapted for radiated emissions testing of electrical devices over frequency range of $10 \mathrm{kHz}$ to $100 \mathrm{MHz}$. Emissions testing using this device is now a provisional test technique for the IEEE Standard Test Method, PAR-1140. Our work in photonic-linked antennas also resulted in a standard, spherical dipole radiator that has found application in characterizing test ranges and chambers and has become a commercial product. 


\section{Magnetic Instruments and Materials Characterization}

\section{Project Leader/Contact:}

Ron Goldfarb, (303) 497-3650, goldfarb@boulder.nist.gov

Approximate staff (FTE):

3 Professionals

Funding sources:

NIST (60\%), Other Agency (10\%), Other (30\%)

Objective:

Develop metrological methods for magnetism and magnetic materials and support measurement needs of magnetic data storage industry:

Constituency:

Magnetic data storage industry

Major current tasks:

- Develop near-field optical techniques for magnetic imaging.

- Investigate nonlinear magneto-optic effects.

- Extend near-field instruments for time-resolved measurements.

Additional information:

A scanning near-field optical microscope (SNOM) which employs magneto-optic Kerr effect (MOKE) contrast has recently been completed at VIST. The instrument holds the promise of directly imaging magnetic domain structures with a resolution approaching $10 \mathrm{~nm}$. A theory to explain images obtained with such as instrument is now being formulated. This instrument is under development in order to address the need in the magnetic data storage industry for a robust, flexible, and readily interpretable magnetic imaging technique that does not require either an expensive ultra-high vacuum environment or lengthy sample preparation methods. A Competence Award has recently been received, part of which is to be used for the extension of MOKE-SNON to time-resolved imaging. By using a femtosecond laser light source for illumination, dynamical magnetıc phenomena may be observed via stroboscopic methods. In addition, nonlinear phenomena such as giant magneto-optic effects observed in second harmonic generation may be investigated for possible diagnostic and data storage applications. 


\section{Laser Radiometry}

\section{Project Leader/Contact:}

Christopher Cromer, (303) 497-5620, cromer@boulder.nist.gov

Approximate staff (FTE):

6 Professionals, 2 Contractors, 1.5 Technicians

Funding sources:

NIST (64\%), Other Agencies (31\%), Other (5\%)

Objective:

Develop measurement methods and standards for characterizing laser sources and detectors used primarily with $\mathrm{cw}$ laser radiation. Develop and maintain measurement services for laser power and energy, optical fiber power, and related parameters (spatial uniformity, linearity, spectral responsivity, etc.).

\section{Constituency:}

Manufacturers and users of lasers for industrial and medical applications; optical communications industry; DoD

\section{Major current tasks:}

- Develop vertical-cavity surface-emitting laser measurement methods and standards.

- Develop and provide measurement services for optical fiber power meters.

- Develop and provide measurement services for laser power and energy detectors.

- Improve accuracy of laser and optical fiber power measurements.

- Develop beam profile measurement capability.

\section{Additional information:}

Accurate characterization of optoelectronic sources and detectors is crucial to the effective development and use of industrial technologies such as lightwave telecommunications, laser-based medical instrumentation, materials processing, photolithography, data storage, and laser safety equipment. Members of the Laser Radiometry Project develop standards and provide measurement services for selected critical parameters intrinsic to optoelectronic sources and detectors, especially the calibration of optical fiber power meters and laser power/energy meters at commonly used wavelengths and power levels. In addition, special test measurements are available for linearity, spectral responsivity, and spatial uniformity of optical power meters and detectors. In support of source characterization, Project members develop measurement methods to evaluate and reduce laser intensity noise, and characterize beam intensity profile and propagation of laser beams. Project members participate in national and international standards committees that are developing standards for laser safety, laser radiation measurements (such as beam profile and pointing stability), and optical-power-related measurements. Project scientists continue to extend and improve source and detector characterizations, including development of low noise, spectrally flat, highly uniform pyroelectric detectors, and tunable-wavelength optical-fiber power measurement systems. 


\section{High-Speed Measurements}

Project Leader/Contact:

Paul Hale, ( 303 ) 497-5367, hale@boulder.nist.gov

Approximate staff (FTE):

4 Professionals, 0.7 Technician, 1 Guest Researcher

Funding sources:

NIST (65\%), Other Agencies (30\%), Other (5\%)

\section{Objective:}

Provide metrology, standards, and measurement services relating to temporal properties of optical sources and detectors used in association with optoelectronic systems.

\section{Constituency:}

Optoelectronic test equipment manufacturers, laser and detector manufacturers, optical communications industry, DoD

\section{Major current tasks:}

- Photoreceiver frequency response

- Photoreceiver impulse response

- Relative intensity noise

- Low level pulsed radiometry

- Excimer laser pulsed measurements

\section{Additional information:}

High-bandwidth measurements are needed to support high-performance systems that take advantage of the potential bandwidth of optical fiber. Systems presently being installed operate at 5 to 10 gigabits per second (Gbit/s) using pure optical time division multiplexing (OTDM), and research is being done on the next generation of OTDM systems at 20 to $40 \mathrm{Gbit} / \mathrm{s}$. Methods are needed to characterize the frequency and impulse response of high-speed sources and detectors to at least the third harmonic of the system modulation rate. Burst mode operation in asynchronous transfer mode networks requires characterization at very low frequencies. Increasingly tight tolerances in both digital and analog systems require frequency response measurements with low uncertainty. Source and detector noise measurements are required to predict low bit error ratios in computer interconnects, high carrier-to-noise ratios in analog systems, and to support erbium-doped fiber amplifier noise figure measurements using electrical noise methods. The high resolution photolithography and corneal sculpting markets require pulsed excimer laser measurements. Intensive use of laser target designators by the armed forces requires traceable low level pulse power and energy calibration standards at $1.06 \mu \mathrm{m}$ and $1.55 \mu \mathrm{m}$. 


\section{Optical Fiber Metrology}

Project Leader/Contact:

Douglas L. Franzen, (303) 497-3346, franzen@boulder.nist.gov

Approximate staff (FTE):

4.5 Professionals, 0.3 Technician

Funding sources:

NIST $(100 \%)$

\section{Objective:}

Develop advanced measurement methods and Standard Reference Materials (SRMs) for optical fibers; interact with standards groups such as the Telecommunications Industry Association to provide a metrology base for the lightwave communications industry.

\section{Constituency:}

Principally the optical communications industry; domestic and international standards groups

\section{Major current tasks:}

- Dimensional metrology for optical fibers

- Dispersion metrology for optical fibers

- Nonlinear properties of fiber

- Optical fiber system/field measurements

- Optical fiber amplifier characterization

\section{Additional information:}

NIST research on measurement methods for optical fiber began in 1976, and has focused on the development and evaluation of measurement techniques for key parameters of interest in product specification and manufacturing. Staff u orh closely with domestic and international industry standards organizations and are frequently called upon to serve as neutral parties in verifying the suitability of proposed standards through interlaborator comparison; over 20 such studies have been conducted to date. In cooperation with the Precision Engıneering Division (see page 18), dimensional metrology and standard reference materials have been developed for optical fiber, fiber coatings, connector ferrules, and ferrule gage wires. The cladding diameter specification of most fiber manufactured in the U.S. is currently traceable to NIST through an SRM developed by the Group. SRMs for fiber chromatic dispersion (zero-dispersion wavelength and slope) and polarization mode dispersion are in the final stages of development. Studies of fiber nonlınearities have demonstrated the close relationship between four-wave mixing efficiency and fiber dispersive properties. NIST staff were among the early developers of optical time domain reflectometr. (OTDR) and low coherence reflectometry and continue to exploit their usefulness in fiber characterization. 


\section{Integrated Optics Metrology}

\section{Project Leader/Contact:}

Matt Young, (303) 497-3223, myoung@boulder.nist.gov

Approximate staff (FTE):

1.5 Professionals, 0.7 Contractor

Funding sources:

NIST (100\%)

\section{Objective:}

Develop advanced measurement methods for integrated optical waveguides. Interact with standards groups to provide a metrology base for the lightwave industry.

\section{Constituency:}

Principally the optical communications industry; domestic and international standards groups

Major current tasks:

- Dimensional metrology

- Low coherence reflectometry

- Mode-field profiles

- Waveguide attenuation

Additional information:

As the optical fiber industry moves toward local area networks and toward fiber to the home, there is increasing need for inexpensive passive components such as splitters. Additionally, such components are needed because long-distance telephony is retrofitting to wavelength division multiplexing. Several companies are manufacturing $1 \times \mathrm{N}$ splitters. There are, however, no standard measurement procedures similar to those for fiber for such parameters as index profile, mode-field diameter, and physical dimensions. Moreover, it is not obvious how to perform the analogous measurements. This project is examining several approaches to measurements of the more critical parameters. 


\section{Fiber and Discrete Components}

Project Leader/Contact:

Sarah L. Gilbert, (303) 497-3120, sgilbert@boulder.nist.gov

Approximate staff (FTE):

2.5 Professionals, 1 Contractor

Funding sources:

NIST $(100 \%)$

Objective:

Develop measurement methods for characterization of optical fiber components and discrete components and develop standards needed by industry for these components.

\section{Constituency:}

Primarily the optical communications industry

Major current tasks:

- Wavelength standards for optical communications

- Metrology for photo-induced Bragg gratings in optical fiber

- Polarization-dependent loss metrology

\section{Additional information:}

Advanced optical communications systems use many different types of optical components to control and modify propagating signals. The Fiber and Discrete Components Project develops measurement technology to characterize these components and understand their limits. One area of research is Bragg gratings in optical fiber; these gratings are good candidates for incorporation into a variety of components. This research includes the design, fabrication, and characterization of fiber gratings and components which contain these gratings. Of particular interest are the long-term stability of fiber gratings and the characteristics of laser sources which incorporate fiber gratings. Another area of research is the study of polarization-dependent loss of components. An artifact polarization-dependent loss standard is being developed to calibrate instruments which measure such loss in optical fiber components. Wavelength standards for the optical fiber communication bands are being developed to support multi-wavelength communication systems. This work involves moderate and high resolution spectroscopy of atoms and molecules in the $1300 \mathrm{~nm}$ and $1550 \mathrm{~nm}$ regions. 


\section{Optical Fiber Sensors}

\section{Project Leader/Contacts:}

Kent B. Rochford, (303) 497-5170, rochford@boulder.nist.gov

Approximate staff (FTE):

3 Professionals, 0.6 Technician, 1.5 Guest Researchers

Funding sources:

NIST (70\%), Other Agency (30\%)

Objective:

Provide metrology to support the optical fiber sensor industry and develop advanced sensing technology for other government and industry laboratories; provide polarization measurements and develop polarization standards for industry; provide optical disc substrate measurements.

\section{Constituency:}

Optical fiber sensor industry, optical communications industry, manufacturers of polarization components and polarization measuring instruments, optical disc replicator and test equipment manufacturers, DoD, DoE, NASA

\section{Major current tasks:}

- Systems-level sensor research and development

- Sensor components research and development

- Standard retarder for polarization analyzer calibration

- Retardance measurement for waveplates and optical discs

\section{Additional information:}

Optical fiber opens the possibility of making sensors that are smaller, lighter, faster, and especially, less susceptible to interference. The Optical Fiber Sensors Project develops measurement methods and standards in support of the optical fiber sensor industry, particularly for optical components used in sensors and new sensor technology, primarily in support of the needs of other government laboratories. One example is the use of the Faraday effect in optical materials. This provides a nonelectrical means of measuring magnetic fields; sensors based on this technology are relatively nonperturbing and immune to electrical interference. Recent work has yielded dramatic improvements in their performance. In addition, fiber sensors have been developed for other measurands, such as electrical current and temperature. Since polarimetric detection techniques are often used, the project has developed considerable expertise in polarization metrology. Several methods for accurately measuring retardance have been developed, and an effort to create a linear retardance artifact standard for calibrating polarization analyzers is nearing completion. More recently, these measurements are being extended to special tests and are applied to the calibration of optical disc-testing equipment. 


\section{Semiconductor Materials and Devices}

\section{Project Leader/Contact:}

David H. Christensen, (303) 497-3354, christen@boulder.nist.gov

Approximate staff (FTE):

5 Professionals, 2 Contractors, 1 Guest Researcher

Funding sources:

NIST (93\%), Other Agency (7\%)

\section{Objective:}

Develop measurement methodology and provide data to support the efficient manufacture of semiconductor optoelectronic devices; provide advanced devices to support research in other parts of NIST.

\section{Constituency:}

Semiconductor optoelectronic device developers and manufacturers

\section{Major current tasks:}

- Characterization of vertical-cavity semiconductor structures

- In-process optical measurement of epitaxial growth

- Vertical-cavity surface-emitting laser metrology

- Devices for advanced metrology and sensing

\section{Additional information:}

The operation of semiconductor optoelectronic devices depends critically on the thickness and composition of hetero-epitaxial layers. To improve measurement accuracy, NIST scientists correlate data from nondestructive techniques, such as x-ray diffractometry, photoluminescence spectroscopy, and reflectance spectroscopy, with that from destructive techniques such as transmission electron microscopy. In situ optical characterization of epitaxial crystal growth is used to improve manufacturing yield and thereby reduce cost. Cross-sectional micro-photoluminescence spectroscopy is being applied to measure defect assisted $\mathrm{Al}-\mathrm{Ga}$ interdiffusion processes and quantum microcavity effects. In support of next-generation optical interconnect, display, and data storage products, Division staff are measuring the properties of arrayed surface-emitting lasers. Ongoing collaborations explore the use of VCSELs in sensing applications. The photon and carrier dynamics in vertical-cavity lasers are characterized using novel ultrafast measurement methods and device structures. Optical characterization methods for group III-nitride materials are being developed to support the manufacture of blue-wavelength optoelectronic components. 
Electronics and Electrical Engineering Laboratory

Optoelectronics Division

Optoelectronic Manufacturing Group, 815.04

\section{Dielectric Materials and Devices}

Project Leader/Contact:

Norman A. Sanford, (303) 497-5239, sanford@bldrdoc.gov

Approximate staff (FTE): 4 Professionals, 2 Contractors

Funding sources:

NIST (63\%), Other Agency (32\%), Other (5\%)

\section{Objective:}

Develop advanced measurement and characterization methods for dielectric waveguide materials and processing procedures for these materials. Develop advanced numerical modeling procedures for solid-state waveguide lasers and nonlinear optical devices.

\section{Constituency:}

Primarily, developers and manufacturers of glass and ferroelectric integrated optical devices Developers and manufacturers of sensor systems, compact solid-state lasers, and nonlinear optical devices and systems

\section{Major current tasks:}

- Characterization and processing of rare-earth-doped silicate and phosphate glass

- Fabrication, characterization and modeling of cw, Q-switched, and mode-locked waveguide lasers

- Characterization and processing of domain-engineered structures in $\mathrm{LiNbO}_{3}$ and $\mathrm{LiTaO}_{3}$

- Measurements of $\mathrm{LiNbO}_{3}$ and $\mathrm{LiTaO}_{3}$ compositional uniformity, doping, and domains

\section{Additional Information:}

NIST is developing measurement methods and acquiring critical materials data to improve the manufacturing of glass, $\mathrm{LiNbO}_{3}$, and $\mathrm{LiTaO}_{3}$ waveguide devices for telecommunications, data storage, and sensor applications. A number of state-of-the-art, rare-earth-doped waveguide lasers have been demonstrated: cw, pulsed, single-frequency, distributed Bragg-reflector, multi-wavelength $(906,1057$, $1328,1358 \mathrm{~nm})$, visible $(540 \mathrm{~nm})$, and tunable lasers. In collaboration with glass and device manufacturers, rare-earth-dopant and ion-exchange parameters are correlated with near-field profile, effective index, loss, AFM measurements, and modeling. This is leading to optimized cw and pulsed solid-state waveguide lasers and optimized waveguide amplifiers. Precise characterization methods of the domain-reversal process in $\mathrm{LiNbO}_{3}$ and $\mathrm{LiTaO}_{3}$ are under development. NIST scientists and their collaborators have developed a means for direct imaging of domain-engineered structures in $\mathrm{LiNbO}_{3}$ using a modification of atomic-force microscopy. Reflection and transmission x-ray topography has been used to examine and characterize bulk and waveguide samples of $\mathrm{LiNbO}_{3}$. Maker fringe analysis has been applied to the mapping of $\mathrm{LiNbO}_{3}$ and $\mathrm{LiTaO}_{3}$ wafers for the examination of compositional uniformity. Device manufacturers have interest in such studies to improve production yields. 


\section{Standard Reference Materials for Fiber and Ferrule Geometry}

\section{Project Leader/Contact:}

Ted Doiron, (301)975-3472, doiron@enh.nist.gov

Approximate staff (FTE):

1 Professional

Funding sources:

NIST $(100 \%)$

\section{Objective:}

Develop standard reference materials (SRMs) for calibrating instruments which measure optical fiber diameter and ferrule geometry.

\section{Constituency:}

Fiber optics industry

\section{Major current tasks:}

- Confirm measuring machine accuracy through national and international comparisons.

- Measure diameter of $126 \mu \mathrm{m}$ steel pin gages.

- Measure diameter and roundness of ferrules.

\section{Additional Information:}

This project is performed in conjunction with the Optoelectronics Division of NIST in Boulder, Colorado (see page 12). The high accuracy micrometer used for these measurements is identical to that codeveloped for calibrating standard reference optical fibers. Both instruments were upgraded in the last year. The SRM for fiber cladding diameter is certified by Division 815.02 in Boulder. Comparisons between various NIST measuring machines and the machine used at NIST/Boulder for the optical fibers have shown excellent agreement on both steel wires and ferrules, generally well within the $0.05 \mu \mathrm{m}$ uncertainty reported on the standard reference fibers. 
Manufacturing Engineering Laboratory

Precision Engineering Division

Mid-Ṡcale \& Complex Form Metrology Group, 821.12, Nano-scale Metrology Group, 821.14

\section{Diode Lasers for Length Measurement}

Project Leader/Contact:

Jack Stone, (301)975-5638, stoneja@enh.nist.gov

Lowell Howard, (301)975-3227, lhoward@enh.nist.gov

Approximate staff (FTE):

1.5 Professionals, 0.5 Technician

Funding sources:

NIST $(100 \%)$

Objective:

Develop new applications for diode lasers to solve problems of length metrology.

Constituency:

Aerospace, precision machining, semiconductor metrology

Major current tasks:

- Absolute Distance Interferometry

- Dynamic Displacement Artifact for nanoscale metrology

\section{Additional information:}

Diode lasers have certain advantages-particularly their ability to be tuned over broad frequency ranges-that may impact length metrology. We are currently developing a diode-laser based system for absolute interferometry intended to overcome limitations of existing techniques. This measurement system will not require a continuously uninterrupted beam, as is needed for displacement interferometry, but will attain the high level of accuracy characteristic of interferometric measurements.

We are also building a Dynamic Displacement Artifact, that employs a broadly tunable diode laser locked to a variable-length cavity to measure displacements of several micrometers with picometer precision. The Dynamic Displacement Artifact will provide length metrology for scanning probe microscopes and for step-height measurement.

In addition, we are working with the Time and Frequency Division of NIST to implement new diode laser sources with particular advantages for length metrology. 


\section{Bacteriorhodopsin for Information Storage and Retrieval}

\section{Project Leader/Contact:}

Howard H. Weetall, (301)975-2628, howard.weetall@nist.gov

Approximate staff (FTE):

2 Professionals, 1 Guest Researcher

Funding sources:

NIST $(100 \%)$

\section{Objective:}

Develop a biological system and device for information storage and retrieval using a light-sensitive protein as the storage agent.

\section{Constituency:}

Data storage, computer, and electronics industries

\section{Major current tasks:}

- Preparation of genetically modified bacteriorhodopsins

- Preparation of chromophore analogs of wild-type and mutant bacteriorhodopsins

- Preparation of monolayer/bilayers of bacteriorhodopsin

- Optical characterization of monolayer/bilayers of wild-type and mutant bacteriorhodopsins and their chromophore analogs

- Chemical treatment or modification to increase life-time of "M" state

- Demonstrate information storage and retrieval using monolayer/bilayers of bacteriorhodopsin

\section{Additional information:}

This project has been underway at NIST for four years. During this time, we have established methods for the chemical treatment of bacteriorhodopsin to increase the time required for the photocycle completion from milliseconds to several minutes. We have additionally learned to prepare films and solgels of bacteriorhodopsin with retention of activity and high spatial resolution. Present studies on the preparation of chromophore analogs have led to the preparation of one blue shifted analog protein with an "M" state decay rate in excess of $120 \mathrm{~min}$. These new derivatives, as well as the native protein, are being subjected to spectral and electro-optical characterization for retention of proton-pumping ability. Methods are being developed to prepare monolayers of bacteriorhodopsin using Langmuir-Blodgett technology and covalent attachment to inorganic surfaces as a first step in producing an information storage system. 


\section{Electronic Structure and Surface Characterization of Oxides}

Project Leader/Contact:

Steven W. Robey, (301)975-2550, srobey@enh.nist.gov

Approximate staff (FTE):

1 Professional

Funding sources:

NIST (100\%)

\section{Objective:}

Develop an understanding of the bulh and surface electronic structure of oxides such as $\mathrm{BaTiO}_{3}$ and $\mathrm{LiNbO}_{3}$, and the influence of surface and interface chemistry and structure on oxide thin-film growth.

\section{Constituency:}

Integrated optoelectronic and ferroelectric device industry, academic research institutions

\section{Major current tasks:}

- Surface characterization (chemical interactions, electronic and atomic structure) of oxides

- Thin-film growth of oxides and buffer layers

- Fundamental studies of the electronic structure of oxide materials

- Development of surface processing necessary for integrating GaAs and nonlinear oxides

\section{Additional information:}

The program involves strong collaborations $u$ th the University of Maryland and Yale University. Major components consist of studies of the surface interface electronic and atomic structures in the growth of oxide thin films, and studies of the electronıc structure of oxides using synchrotron radiation.

Surface/interface sensitive techniques are used to provide in situ characterization of electronic structure, surface chemistry, and microstructure for thin films produced by MBE and pulsed laser deposition. Important information on surface morpholog! is provided by ex situ atomic force microscopy. Ultraviolet photoelectron spectroscopy is used to study the electronic structure in perovskite-based oxides. Current emphases include: (1) integration of oxides with $\mathrm{GaAs}$ for optoelectronic applications (this work is currently focussed on the grouth of buffer layers for the integration of oxides such as $\mathrm{BaTiO}_{3}$ and $\mathrm{LiNbO}_{3}$, with $\mathrm{GaAs}$ ): (2) studies of correlations between composition-induced changes in transport and magnetic properties and electronic structure in perovskite-based oxides. 


\section{Vibrationally-Resolved Near-Field-Scanning Optical Microscopy}

Project Leader/Contact:

Richard Cavanagh, (301) 975-2368, richard.cavanagh@nist.gov

Approximate staff (FTE):

2 Professionals

\section{Funding sources:}

NIST (100\%)

\section{Objective:}

Develop advanced measurement methods based on near-field-scanning optical microscopy for the characterization of the chemical composition of materials with submicrometer spatial resolution.

\section{Constituency:}

Chemical, coatings, biotechnology, and semiconductor industries

Major current tasks:

- Develop high brightness, tunable infrared (IR) laser sources appropriate for coupling to nearfield probes.

- Develop near-field probe structures appropriate for 2 to $10 \mu \mathrm{m}$ radiation.

- Determine the interaction mechanisms between vibrationally-resonant evanescent IR radiation and samples that give rise to image contrast.

\section{Additional information:}

In 1996, NIST launched a program in the development of chemical probes with high spatial resolution. The initial program focus is the development of a vibrationally-resolved, scanning IR microscope with spatial resolution better than $800 \mathrm{~nm}$ and spectral coverage from 2.5 to $10 \mu \mathrm{m}$. IR spectroscopy is a nondestructive technique that can provide information on the identity and orientation of chemical functional groups. The instrument will be applied to the characterization of nanostructured materials including heterogeneous catalysts, organic and inorganic thin films, and biological systems. 


\section{Fiber and Integrated Optic Chemical Sensors}

Project Leader/Contact:

Steven Choquette, (301)975-3096, steven.choquette@nist.gov

Approximate staff (FTE):

2 Professionals, 0.3 Student

Funding sources:

NIST (100\%)

Objective:

Develop integrated- and fiber-optic sensors for clinical, environmental, and process analytical applications.

\section{Constituency:}

Chemical, environmental, and medical sensor industries

Major current tasks:

- Develop planar waveguides suitable for visible and NIR wavelengths.

- Develop analytical strategies for chemical sensors.

- Develop combined electrochemical/optical Mach Zehnder sensors.

\section{Additional information:}

We have been involved in planar waveguide chemical sensor research since 1988. Our group has demonstrated planar waveguide sensors for attenuation-based immunoassay schemes, radiation dosimetry, and pesticide analysis. Our current research involves the use of inexpensive waveguide interferometer schemes, combined with electrochemistry, to provide additional selectivity and sensitivity for chemical measurements. In addition, we are working on embossable grating couplers for reproducible and efficient source-to-waveguide coupling. 


\section{Extreme Ultraviolet (EUV) Optics Development and Characterization}

\section{Project Leader/Contact:}

Tom Lucatorto, (301)975-3734,toml@enh.nist.gov

Approximate staff (FTE):

2 Professionals

Funding sources:

NIST $(100 \%)$

\section{Objective:}

Contribute to the development of diffraction-limited EUV optics; provide accurate measurements of properties of EUV optics and optical șstems such as reflectance, transmittance, image quality, and dosimetry.

\section{Constituency:}

Microlithography industry, precision optics industry, $\mathrm{x}$-ray microscopy community

Major current tasks:

- Characterize reflectance of EUV' multilayer mirrors.

- Develop multilayer coating techniques for improved EUV performance.

\section{Additional information:}

The program is centered around the development of multilayered thin film structures for producing high reflectance EUV optics and for figuring those optics to nanometer accuracy in order to achieve diffraction-limited imaging at EUV wavelengths. We have the capability to produce graded, ion-beam polished, multilayer structures by both ion sputtering and thermal evaporation. Our EUV optics characterization instrumentation has the capability to provide high resolution reflectivity maps of mirrors as large as $35 \mathrm{~cm}$ in diameter (and $40 \mathrm{hg}$ in mass) such as will be required for EUV lithography, and to perform transmission and dosimetr. measurements in the range 2.5 to $40 \mathrm{~nm}$. Measurements of figure and finish of these optics to subnanometer accuracy are provided through a collaborative arrangement with the Precision Engineering Division $\mathrm{He}$ uelcome collaborations with any outside institutions pursuing the development and application of ELV optics. 
Physics Laboratory

Electron and Optical Physics Division

Far Ultraviolet Physics Group, 841.02

\section{Vacuum Ultraviolet (VUV) Detector Radiometry}

Project Leader/Contact:

L. R. Canfield, (301)975-3728, canfield@nist.gov

Approximate staff (FTE):

2 Professionals

Funding sources:

NIST (87\%), Other Agency (6\%), Other (7\%)

Objective:

(1) Develop methods for the absolute calibration of detectors in the deep ultraviolet

(DUV)/VUV/soft x-ray spectral region including the development of methods of transfer radiometry from the absolute SURF II synchrotron radiation source to transfer standard detectors; (2) develop transfer standard detectors and establish for them a calibration and supply service.

\section{Constituency:}

Industrial radiometry service providers, plasma diagnostics community, atmospheric research community, space research community

Major current tasks:

- Calibrate working and transfer standard detectors.

- Develop solid state transfer standard detectors.

- Develop radiometric transfer calibration methods and systems.

\section{Additional information:}

The program has evolved since the early 1970's with expansion of the spectral coverage improvements in the radiometric characteristics of detectors and the development of the SURF II storage ring source as an absolute radiometric source. SURF II is now used as the fundamental standard for absolute detector calibration in the DUV and shorter wavelengths to $120 \mathrm{~nm}$. NIST staff routinely serve as consultants for researcher professionals in other federal agencies, industry, and academia, offering a wide range of detector calibration services to meet their far UV radiometric needs. Several transfer standard detector types are routinely available with absolute calibrations covering the spectral region $5 \mathrm{~nm}$ to $254 \mathrm{~nm}$. Improved technologies are actively investigated and have led to the recent collaborative development of semiconductor detectors, which are now available as stable transfer standards over the entire range covered. In addition to these routine detector calibrations, calibration of special detectors and filters are occasionally conducted for interested parties. NIST is in the process of upgrading SURF II to SURF III, which will be a storage ring of greatly improved radiometric accuracy. SURF III will serve NIST as a national standard of radiometric flux from the far infrared to the soft x-ray region. 


\section{Magnetic Characterization by Confocal MOKE Imaging}

Project Leader/Contact:

John Unguris, (301) 975-3712, unguris@epg.nist.gov

Approximate staff (FTE):

0.8 Professional

Funding sources:

NIST (85\%), Other Agency (15\%)

Objective:

Develop instrumentation for optically imaging the magnetic domain structure of ultrathin magnetic films and multilayers.

\section{Constituency:}

NIST, federal, and university research labs, and magnetic device manufacturers

\section{Major current tasks:}

- Measure magnetic coupling strengths in magnetic multilayers.

- Image the field domain structure of thin magnetic films on textured substrates.

- Improve the stability of the microscope illumination system.

\section{Additional information:}

A confocal optical microscope with magnetic contrast derived from the magneto-optic Kerr effect (MOKE) has recently been developed. The microscope combines the magnetic domain imaging capabilities of conventional MOKE microscopy with the surface sensitivity of grazing angle, nonimaging, laser-based MOKE. Surface sensitivity is necessary because most of the next generation of miniature magnetic devices, such as recording heads, recording media and sensors, will use ultrathin ( 0 to $10 \mathrm{~nm}$ ) magnetic films. The microscope is currently being used to measure the magnetic exchange coupling strengths between ultrathin magnetic films separated by nonmagnetic interlayers. 
Physics Laboratory

Electron and Optical Physics Division

Electron Physics Group, 841.03

\section{Characterization of Materials by Optical Emission in Scanning Tunneling Microscopy (STM)}

Project Leader/Contact:

Daniel T. Pierce (301) 975-3711, pierce@epg.nist.gov

Approximate staff (FTE):

1 Professional

Funding sources:

NIST (80\%), Other Agency (20\%)

Objective:

Develop techniques to characterize atomic scale properties of metals and semiconductor surfaces and thin films by measuring photon emission in scanning tunneling microscopy.

\section{Constituency:}

Semiconductor and magnetic device manufacturers, research laboratories

Major current tasks:

- Assess feasibility of obtaining magnetic contrast in the STM from tunneling induced photon emission.

- Add imaging spectrograph to STM for wavelength analysis of emitted photons.

- Develop low temperature STM for high resolution measurements of optical emission from semiconductor heterostructures, quantum wells and quantum wires.

\section{Additional information:}

Scanning tunneling microscopy has proven in the last decade to be a very powerful technique for investigating the local atomic scale properties of materials. Recently, measurements have been made of photons stimulated by the tunneling electrons. This adds a new dimension to the STM and offers the potential of obtaining local optical information about electronic materials on an atomic scale. Electronic properties of quantum wells, wires, or dots can be investigated with nanometer resolution using this high resolution luminescence spectroscopy. Quantum well wires, which are expected to be useful for lasers and modulators, can be probed individually for optically active defects by scanning tunneling luminescence. Instead of an averaged optical measurement of multiple quantum dots using a macroscopic optical probe, the spectrum of an individual quantum dot can be measured thereby allowing an investigation of the influence of size, shape, and defects. The spectrum of energy levels in nanoscopic structures resulting from quantum confinement can be investigated. If photon emission in the STM is successful in producing magnetic contrast, it will be also possible to measure magnetic microstructure with unprecedented resolution and in the range required for characterizing next generation hard disk magnetic storage media. 


\section{Nanofabrication with Atom Optics}

Project Leader/Contact:

Jabez McClelland, (301) 975-3721, jabez@epg.nist.gov

Approximate staff (FTE):

3.2 Professionals

Funding sources:

NIST $(100 \%)$

Objective:

Explore optical methods of controlling the motion of atoms, including laser focusing, with the goal of developing new methods for fabricating nanoscale devices with feature size as small as $10 \mathrm{~nm}$ and emphasis on electronic and magnetic device fabrication.

\section{Constituency:}

Optoelectronic device fabrication industry; microfabrication industry, especially those involved in nanoscale standards; data storage industry

Major current tasks:

- Pursue further developments in laser-focused atomic deposition in a standing wave, including increasing resolution and reducing background.

- Develop techniques for generating arbitrary patterns with laser-controlled atomic deposition.

- Examine methods for transferring patterns to surfaces using metastable rare gas atoms.

\section{Additional information:}

This project has already demonstrated the capability of fabricating lines and dots on a surface with dimensions of tens of nanometers using laser-focused atomic deposition of chromium in a standing wave. The process is inherently parallel, meaning that large areas of a surface can be covered with highresolution features all at once. Thus it has potential advantages over electron or ion beam lithography (because it is a parallel process) or optical lithography (because the resolution is higher). Possible applications range from eventual use in fabrication of microelectronic devices, to patterning of magnetic media for discretized recording. Especially significant is the ability to take advantage of the long-range coherence of a laser standing wave to fabricate very large arrays of identical nanoscale features. This ability is particularly relevant to the optoelectronics industry. 


\section{Atomic Data for High Intensity Lighting}

\section{Project Leader/Contact:}

Craig Sansonetti, (301)975-3223, sans@enh.nist.gov

Approximate staff (FTE):

2 Professionals

Funding sources:

NIST $(100 \%)$

\section{Objective:}

The objective of the project is to develop basic atomic data needed by the lighting industry for design and modeling of high intensity discharge lamps.

\section{Constituency:}

High intensity discharge lamps are produced by all major lamp manufacturers. Atomic data required to improve these sources is an infrastructural contribution to the knowledge base on which high efficiency lighting rests from which all manufacturers will benefit.

\section{Major current tasks:}

- Install Fourier transform spectrometer (FTS) as primary instrument for this program.

- Develop sources to excite the spectra of neutral and low ionization stages of rare earth atoms.

- Develop methods for radiometric calibration of the FTS spectra.

- Observe rare earth spectra (first candidate species is thulium) to measure accurate wavelengths and intensities.

- Analyze the spectra to obtain accurate energy levels and branching ratios for use in lamp modeling.

- Publish research results.

\section{Additional information:}

Rare earth elements are used in high intensity discharge lamps to improve their efficiency and color rendering properties. Lamp manufacturers are currently developing computer modeling techniques to predict the operating characteristics of such lamps. These computer codes may be able to replace costly and time consuming trial-and-error procedures for developing improved lighting systems. A primary limitation in the use of the computer codes is lack of fundamental atomic data for the atoms and ions of interest. NIST research in this area is focused on atomic data needs that are directly applicable to design and modeling of high intensity discharge lamps. Our principal research activity will be observation of spectra to obtain wavelengths, energy levels, and branching ratios for rare earth atoms and ions. These data can be combined with lifetimes being measured in university laboratories to obtain oscillator strengths for large numbers of transitions. 


\section{Nanoscale Surface Modification for Optical Components}

\section{Project Leader/Contact:}

John Gillaspy, (301) 975-3236, Gillaspy@enh.nist.gov

Approximate staff (FTE):

2.5 Professionals

Funding sources:

NIST $(100 \%)$

Objective:

Explore novel surface modifications that occur under bombardment with very highly charged ions. Identify and develop applications, with particular attention to nanoscale optical and electronic components.

\section{Constituency:}

The microelectronics and photonics manufacturing sector, universities, and government agencies. Present work is ongoing in conjunction with a Cooperative Research and Development Agreement with the Advanced Lithography Group, a consortium which includes industry, universities, and the U.S. Government.

\section{Major current tasks:}

- Produce and characterize directed beams of highly charged ions with a compact laboratory device.

- Bombard a variety of surfaces and characterize the damage.

- Attempt to fabricate a nanoscale diode using highly charged ions.

\section{Additional information:}

The recent development of compact and affordable devices which can produce ions with extreme charge states opens up the possibility of new techniques for modifying surfaces. Qualitatively new underlying mechanisms have already been reported for charges above 30+. The improved fabrication of small-scale photonic devices or the development of novel surface etching methods for optical components are two examples of areas that may benefit from this work. 
Physics Laboratory

Atomic Physics Division

Laser Cooling and Trapped Atoms Group, 842.04

\section{Atomic Photonics}

Project Leader/Contact:

William D. Phillips, (301) 975-6554, wphillips@nist.gov

Approximate staff (FTE):

1 Professional, 1 Guest Researcher

Funding sources:

NIST (100\%)

\section{Objective:}

Develop laser cooling and trapping techniques for manipulation of matter especially for the creation, fabrication and evaluation of optical materials and devices.

\section{Constituency:}

Atom optical manipulation has application in the creation of new-generation atomic time and frequency standards, as well as to highly parallel nanolithography for manufacturing. Manipulation of larger objects has potential application in micromechanical and optical assemblies, for medical laboratory testing and environmental sensing. The techniques being developed here are used in research labs across the U.S. and around the world for scientific and technological purposes.

\section{Major current tasks:}

- Develop atom optical devices for atomic beams

- Develop techniques for atom exposure of resists

- Optical manipulation of objects for microassembly and microanalysis

- Creation of novel optical materials with cold atoms

\section{Additional information:}

We are studying the basic physics of manipulating atoms to produce atom optical devices useful for measurement and manufacturing, such as atom interferometers for inertial sensing and atom lenses and holograms for nanofabrication. We have pioneered the exposure of novel self assembled monolayer (SAMs) resists using laser manipulated atomic beams. Such techniques may have a significant impact for the quantum electronics and photonics manufacturing industry. We are applying optical manipulation of micrometer-sized objects to the characterization and development of biosensors. We have applied this optical tweezer technology to the study of virus-cell attachment. We are also investigating the application of this technique for microassembly and novel forms of scanning microscopy. The ability to create optical lattices and atomic gases at ultra-low temperatures provides new opportunities for exploiting the optical characteristics of such media. Periodic media such as optical lattices offers the possibility of photonic bandgaps, and cold gases allow coherent nonlinear effects such as coherence induced transparency and amplification or lasing without inversion to be achieved without resorting to high pump powers to overcome Doppler broadening. We are investigating a number of such possibilities in our laboratory. We are also investigating the use of the quantum collective behavior of cooled and trapped atoms (Bose-Einstein condensations) to make an "atom laser," a deBroglie-wave analog of an optical laser. 


\section{Spectral Radiometry}

Project Leader/Contact:

Robert Saunders, (301) 975-2355,Rds@enh.nist.gov

Approximate staff (FTE):

6 Professionals, 1 Student

Funding Resources:

NIST (50\%), Other Agency (45\%), Other (6\%)

Objective:

Maintain and disseminate the national scales of optical radiation temperature, spectral radiance, spectral irradiance.

\section{Constituency:}

Major current tasks:

- Establish radiometric scales based on the high accuracy cryogenic radiometer (HACR).

- Resolve the temperature scale discrepancy.

- Setup a radiometric beamline on SURF II for uv source and detector characterization

- Develop calibration techniques for cross-calibrating satellites

- Establish a UV-B monitoring network with USDA and EPA.

Additional information:

Measurements of the optical properties of material in an ambient background are performed in the Optical Temperature and Source Group. These properties include transmittance and reflectance as a function of angle (BRDF). The group is heavily involved with the CCPR of the BIPM, Council on Optical Radiation (CORM), and various ASTM committees. The group is involved with other agencies in solving radiometric measurement problems associated with environmental monitoring. A monitoring station has been assembled on the roof of the Physics Building to measure the terrestrial UV solar irradiance. The data from this station are linked to universities and other agency monitoring sites and serves as a baseline measurement station. The Optical Temperature and Source Group has been involved in thermal imaging and has developed a number of large area blackbodies used to characterize thermal imagers and is developing techniques to characterize focal plane arrays of various kinds. 
Physics Laboratory

Optical Technology Division

Optical Properties and Infrared Technology Group, 844.04

\section{Infrared Metrology}

Project Leader/Contact:

Raju Datla, (301) 975-2131, raju.datla@nist.gov

Approximate staff (FTE):

7 Professionals, 1 Contractor

Funding sources:

NIST (30\%), Other Agency (70\%)

Objective:

Develop advanced measurement methods, transfer standards, Standard Reference Materials (SRMs) and data on infrared optical properties of materials and devices, and support industry standardization of measurement practice through calibration services.

\section{Constituency:}

Major current tasks:

- Development of high optical density filter SRMs for 2 to $25 \mu \mathrm{m}$

- Development of diffuse reflectance SRMs for the infrared

- Measurements of optical properties of materials in the infrared

- Low Background Infrared (LBIR) facility for infrared source and detector calibrations

- Infrared transfer standard detector development

- Advanced radiometry using correlated photons

\section{Additional information:}

The Optical Properties and Infrared Technology Group was established at NIST in 1992 to focus research on measurement methods and development of standards for infrared metrology at cryogenic and room temperature backgrounds. Staff in the group work closely with academia, DoD contractors and other agency experts to serve the national needs for infrared measurements. An absolute cryogenic radiometer (ACR) has been built and characterized as a national standard for low background infrared radiation measurements. The NIST Low Background Infrared (LBIR) calibration facility, which houses the ACR in a cryogenic chamber, has been serving customers since 1989 , providing calibrations for radiance temperature measurements of blackbodies. Spectral capability to provide emissivity, reflectance and transmittance of materials at cryogenic temperatures is being added to this facility. An ambient detector calibration facility has been built to characterize single detectors and arrays and to provide transfer standard detectors for industrial needs. An active program to build new transfer standards using high- $T_{c}$ superconductor material properties is underway. State-of-the-art spectrophotometry equipment has been built to measure optical properties of materials. Advanced radiometry concepts using correlated photons generated in the down conversion process in nonliinear crystals are being explored for use as a source and detector calibration scheme. 


\section{Detector Metrology}

Project Leader/Contact:

Albert C.Parr, (301) 975-2316, albert.parr@nist.gov

Approximate staff (FTE):

14 Professionals, 3 Students, 1 Guest Researcher

Funding sources:

NIST (58\%), Other Agency (35\%), Other (7\%)

\section{Objective:}

Perform research and advanced metrology for optical radiation measurements in the UV, visible, and infrared spectra regions. Provide high accuracy detector calibrations to support industry and the Divisions optical metrology.

\section{Constituency:}

Industrial, military, and government activities

\section{Major current tasks:}

- High accuracy optical power measurements (optical watts)

- Photometric and colorimetric research and metrology

- Research and metrology in optical scattering from materials

- Deep UV metrology for semiconductor photolithography

- R\&D for state-of-the-art optical sensors

- High-accuracy metrology of optical apertures

- Photometry and colorimetric metrology

- Detector calibration services (200 nm to $20 \mu \mathrm{m})$

- Research in near-field scanning optical microscopy

\section{Additional information:}

The primary mission for the group is to provide high quality optical measurements for optical power. These measurements support optical metrology within the division and for calibration services provided to industrial, academic, and military customers, and form the basis of the division's photometric, spectroradiometric, and radiation temperature scales. These services support a wide range of activities including UV semiconductor photolithography, environmental remote sensing, display colorimetry, lighting efficiency, nondestructive testing, and DoD applications such as night vision scopes and smart weapons systems. 


\section{Optical Scattering Metrology}

\section{Project Leader/Contact:}

Thomas Germer, (301)975-2876, germer@enh.nist.gov

Approximate staff (FTE):

1.6 Professionals

\section{Funding sources:}

NIST (100\%)

\section{Objective:}

Develop advanced metrology techniques and standard artifacts for detection and analysis of particles, defects, and surface microroughness on a variety of optical quality surfaces, including bare silicon wafers. More fundamentally, to study the light-scattering interactions between surface features and incident electromagnetic waves.

\section{Constituency:}

Semiconductor metrology equipment manufacturers; optical materials manufacturers

\section{Major current tasks:}

- Correlate bidirectional reflectance distribution function (BRDF) measurements of silicon wafers exhibiting different levels of optical haze with haze measurements carried out with commercial scanning surface inspection sy'stems

- Develop models for optical scantering from particles, defects, and surface microroughness that increase the utility of optical scattering measurements in process control environments.

- Investigate optical scattering from particles on Si surfaces, correlating scattering distributions with particle size and composition.

- Evaluate optical scattering as a metrological tool to assess uniformity of periodic structures such as found on CDS, patterned photoresists, and deposited lines on semiconductors.

\section{Additional information:}

The BRDF quantifies the angular distribution of light scattered from a surface as a function of wavelength, polarization state, and angle of incidence. BRDF measurement is useful for evaluating elements contained within optical systems that require the minimization of scattered light, and it is also effective for the characterization of materials and surfaces in control and inspection processes in manufacturing settings. The Goniometric Optical Scatter Instrument (GOSI) at NIST employs lasers to achieve a wide dynamic range and high angular accuracy and precision, well-suited for studying lowscatter surfaces such as silicon wafers and optically smooth mirrors. GOSI achieves a dynamic range of 15 orders of magnitude with a noise lev el of $10^{-9} \mathrm{sr}^{-1}$ determined by Rayleigh scattering from air molecules, and the scatter direction can be measured with an angular resolution of $0.02^{\circ}$. GOSI is housed in a class 10 clean area to maintain sample integrity. 


\section{Near-Field Optics and Microscopy for Nanoscale Optical Characterization}

\section{Project Leader/Contact:}

Lori Goldner, (301)975-3792, lori@bruce.nist.gov

Approximate staff (FTE):

1.5 Professional

\section{Funding sources:}

NIST $(100 \%)$

\section{Objective:}

Develop near-field scanning optical microscopy (NSOM) as a metrological tool for nanometer-scale optical measurements.

\section{Constituency:}

Researchers and developers of photonic technologies; photonic and semiconductor metrology equipment manufacturers

\section{Major current tasks:}

- Quantify contrast mechanisms in NSOM in various applications, including near-field imaging of photonic structures, latent structures in photoresists, and microcrystalline domains in photonic materials.

- Develop methods to determine resolution of near field optical microscopies.

\section{Additional information:}

NIST is developing NSOM as a quantitative technique for noninvasive optical measurements on previously inaccessible length scales. So far, lateral resolution of the order $20 \mathrm{~nm}$ has been achieved with this technique, and vertical resolution of less than $1 \mathrm{~nm}$ may be possible. NIST is building wellcharacterized microscopes and small light sources, working on methods to determine the resolution of commercially available near-field microscopes, and expanding the capabilities of near-field optics through development of new probes and contrast mechanisms. A significant component of the effort is aimed at understanding the mechanisms that generate contrast in different materials, and modeling the fields around small light sources as they interact with various materials and surface features.

Applications in optoelectronics include mapping of optical properties of nanostructured materials, characterization of the near-field properties of photonic structures, optical data storage, and assessment of nanometer-scale particulate or subsurface defects on optical and semiconductor surfaces. 


\section{Applications of Femtosecond Terahertz Spectroscopy}

\section{Project Leader/Contact:}

Edwin J. Heilweil, (301) 975-2370, ejh@tiber.nist.gov

Approximate staff (FTE):

1.5 Professionals

Funding sources:

NIST (100\%)

\section{Objective:}

Measure electron and phonon dynamics and related optical properties of semiconductors, quantum well structures and optical materials using ultrashort pulsed far infrared ( $\mathrm{THz}$ ) laser techniques.

\section{Constituency:}

Researchers and developers of photonic technologies

\section{Major current tasks:}

- Develop a broadband THz laser system producing subpicosecond pulses, and detection systems to make accurate time-resolved amplitude and phase measurements on the $\mathrm{THz}$ pulses as they interact with samples.

- Determine linear and nonlinear indices of refraction in the $\mathrm{THz}$ range for liquids, solids, and semiconductor multilayer structures.

- Perform time-resolved measurements of electron and phonon coupling phenomena in optical materials and structures.

\section{Additional information:}

NIST is developing a femtosecond laser source (pulses of less than 100 fs duration composed of frequencies below $5 \mathrm{THz}$ ) to measure indices of refraction, carrier dynamics, and other transport properties of photonic materials and structures. Two detection methods (Fourier transform interferrometry and phase-sensitive gated detection) will be used to extract this information. The effort will provide data for materials and device developers, and for improving fundamental understanding of the underlying physical and chemical phenomena. 


\section{Nonlinear Optical Diagnostics of Interfaces and Thin Films}

\section{Project Leader/Contact:}

John Stephenson, (301) 975-2372, jcs@enh.nist.gov

Lee Richter, (301)975-4152, lrichter@enh.nist.gov

Approximate staff (FTE):

3 Professionals

Funding sources:

NIST (78\%), Other (22\%)

\section{Objective:}

Develop advanced measurement methods based on nonlinear optical techniques for the characterization of thin films and their interfaces. Acquire Standard Data for the nonlinear response of thin films and interfaces.

\section{Constituency:}

Semiconductor metrology equipment manufacturers

\section{Major current tasks:}

- Determination of geometric and electronic structures of semiconductor/semiconductor, semiconductor/insulator, and semiconductor/metal interfaces

- Real-time characterization of carrier/trap dynamics at thin-film interfaces

- Determination of surface geometric and chemical structures of organic thin films, including self-assembled monolayers and liquid crystal alignment layers

\section{Additional information:}

NIST has two tunable ultrafast (pulse duration less than $100 \mathrm{fs}$ ) laser systems and associated UHV deposition chambers dedicated to the characterization of the second order nonlinear response of interfaces and thin films. Nonlinear optical (NLO) techniques, made possible by recent advances in laser technology, are excellent nondestructive, in situ diagnostics with unique advantages over traditional linear optical techniques. NLO techniques have greater structural sensitivity than linear techniques, and can be more surface sensitive. Examples of recent studies include: calibration of the nonlinear response of GaAs surfaces arising from the surface space charge field, and use of that calibration to characterize the dynamics of optically injected carriers; optical determination of the orientation of twinned and aligned $\mathrm{Si} / \mathrm{CoSi}_{2}$ interfaces; and characterization of alignment layers for liquid crystal fabrication.

This program is collaborative with the Chemical Sciences and Technology Laboratory, Surface and Microanalysis Science Division, Surface Dynamical Processes Group, 837.03. Staffing and funding levels reflect the combined program. 


\section{Optical Frequency Measurements}

\section{Project Leader/Contact:}

Leo Hollberg, (303) 497-5770, hollberg@bldrdoc.gov

Approximate staff (FTE): 5.2 Professionals, 7.0 Contractors/Postdocs/Students

Funding sources: NIST (98\%), Other Agency (2\%)

\section{Objectives:}

Develop methods for measuring and synthesizing optical signals of narrow linewidth and high stability.

Develop optical frequency standards based on accurately measured frequencies in support of length metrology and spectroscopic analysis. Transfer program-developed, measurement-and-standards technology to industry as opportunities arise.

\section{Constituency:}

Laser and optical component manufacturers; division programs involved in developing advanced atomic frequency standards; other NIST divisions requiring stable, tunable, narrow-linewidth optical sources; other government agencies

\section{Major current tasks:}

- Accurate measurement of optical frequencies

- Development of high-precision semiconductor laser systems

- Simplification of laser systems for frequency standards

- Noise characterization of lasers and related systems

- Wavelength metrology based on optical frequency measurement

- Extending the spectral coverage of diode and solid state lasers throughout the spectrum

- Ultra-sensitive detection of atoms and molecules

- Application of diode lasers to industrial and scientific problems

- Development of semiconductor optical amplifiers

- Study of lasing without population inversion

\section{Additional Information:}

This group traces its origins to division efforts to develop narrow-linewidth diode lasers needed for manipulating and cooling atoms in current and future frequency standards. Such optical sources are also needed to support wavelength standards based on stable spectral features of certain atoms. In developing this program to its current level, NIST management agreed that work on narrow-linewidth, tunable optical sources should not be dispersed, but should be focussed in one group to achieve critical mass. The program has maintained strong ties with industry through CRADA's and work supporting ATP projects. NIST-developed technology transferred under a CRADA has resulted in the introduction of a new line of commercial diodelaser systems. Applications of the group's metrology work extend will beyond the traditional mission of the division to areas such as chemical analysis using laser-spectroscopy methods. The division works closely with the Precision Engineering Division of NIST in the application of optical frequency measurements to length metrology (which is the responsibility of that division). 


\section{Ultrashort Laser Pulse Interactions with Materials}

\section{Project Leader:}

Tracy S. Clement, (303) 492-8198, clementt@jila.colorado.edu

Approximate staff (FTE):

2 Professionals, 2 Students

Funding sources:

NIST $(90 \%)$, OA $(10 \%)$

\section{Objective:}

Develop techniques to study ultrafast and nonlinear processes by measuring, with femtosecond resolution, the intensity and phase evolution of ultrashort laser pulses after interaction with materials.

\section{Constituency:}

Materials processing, laser-produced plasmas, other government and industry laboratories

Major current tasks:

- Determine resolution of phase change measurements/accuracy of technique

- Measure nonlinear properties of optical materials

- Study temporal evolution of electron density in strong-field ionization

\section{Additional Information:}

Recently developed techniques to measure the full electric field (both amplitude and phase) of a single ultrashort laser pulse provide a new way to study, with femtosecond resolution, various aspects of the interaction of light with materials. NIST scientists are currently developing a research program to extend the use of these diagnostics to areas such as materials characterization and ultrafast dynamic spectroscopy. Basic properties of electronic and optoelectronic materials, such as dispersion, nonlinear refraction, two-photon absorption, as well as higher order nonlinear properties, can be determined by measurement of the change of the phase of an optical pulse after the pulse traverses a given material. A novel dynamic spectroscopic technique is being developed to study ultrafast processes in gaseous, liquid, and solid-state materials. In ultrafast pump-probe experiments, including phase information along with the intensity in a single shot can enhance a wide range of ultrafast experiments. 


\section{Near Field Optical Microscopy}

Project Leader/Contact:

Stephen R. Leone, (303) 492-5128, srl@jila.colorado.edu

Approximate staff (FTE):

3.5 Professionals

Funding sources:

NIST (70\%), Other Agency (30\%)

Objective:

Develop measurement methodology for optical probing of nanometer scale structures with spectral resolution and time contrast, as well as use of optical near-fields for atom guiding

Constituency:

Semiconductor industry, interferometry, biomedical analysis, DoD, NASA, DoE

Major current tasks:

- Semiconductor defect analysis

- Molecular sensing

- Gyroscope and metrology development

\section{Additional information:}

Near-field optical microscopy is a technique that permits nanoscale measurements with optical interrogation and spectral resolution. A wide variety of applications are possible, including semiconductor characterization, active manipulation of nanoscale features, and biological imaging. In this project, time contrast methods and spectral resolution techniques are developed for applications in semiconductors and molecular sensing. The near-field light is also used to transport atoms through hollow optical fibers, to create atom interferometers and gyroscopes. In recent work, the optical field is enhanced by metallic nanocolumns, and pulsed lasers will be used to probe spatially resolved photoluminescence GaAs materials. Atoms have been guided through hollow optical fibers with excellent efficiency. 


\section{Optical Coherent Control}

Project Leader:

Stephen R. Leone, (303) 492-5128, srl@jila.colorado.edu

Approximate Staff:

2.5 Professionals

Funding sources:

NIST (20\%), Other Agency (80\%)

\section{Objective:}

To produce designed optical pulses of ultrafast lasers to manipulate molecular processes and materials

Constituency:

Materials processing, optical gratings. fiber optics, semiconductor molecular electronics

Major current tasks:

- Generate pulse trains of ultrashor pulses

- Produce spectral frequency mashs for pulse shaping

- Apply designer pulses to molecular manipulation

Additional information:

Ultrafast pulses with designed frequency and time patterns permit coherent control and manipulation of materials. Such pulses have numerous applications in optical fiber manipulation and communications. In this project, ultrafast pulses are used to study coherent control dynamics in molecules. Amplitude masks are applied to spectrally dispersed pulses to create selective frequencies for coherent excitation. With periodic masks, pulse trains are created $w$ ith terahertz repetition frequency. Such pulses are being used to control vibrational and rotational coherence amplitudes of molecules. 


\section{Optoelectronic Crystals: Linear and Nonlinear Optical Interactions}

\section{Project Leader/Contact:}

Bruce Steiner, (301) 975-5977, bruce.steiner@nist.gov

Approximate staff (FTE):

3 Professionals

Funding sources:

NIST $(100 \%)$

\section{Objective:}

Observe and evaluate disorder in high quality optoelectronic single crystals and its interaction with electromagnetic fields.

\section{Constituency:}

Optical information processing component and systems developers; growers of optoelectronic crystals, both monolithic and layered; academic and governmental researchers investigating related areas

Major current tasks:

- Development of insight into the role of crystalline order in the determination of the properties of optoelectronic crystals

- Control of the principal sources of disorder in these crystals

- High resolution monochromatic synchrotron $\mathrm{x}$-radiation diffraction imaging of optoelectronic crystals, both monolithic and layered

- X-ray diffraction imaging of photorefractive gratings

- Photorefractive grating fixing

- Mathematic modeling of these processes

\section{Additional information:}

Crystals currently under study include: $\mathrm{SBN}, \mathrm{BaTiO}_{3}, \mathrm{LiNbO}_{3}, \mathrm{III}-\mathrm{Vs}, \mathrm{HgI}{ }_{2}$, II-VIs, several of these in monolithic and layered forms. Collaborators include U.S. industry, universities, and government agencies. 


\section{Wide-Bandgap Semiconductors for Short-Wavelength Photonic Devices}

\section{Project Leader/Contact:}

Lawrence H. Robins, (301) 975-5263, lawrence.robins@nist.gov

Approximate staff (FTE):

1 Professional

Funding sources:

NIST (100\%)

\section{Objective:}

To provide critical data on the optical and optoelectronic properties, especially defect-related properties, of wide-bandgap semiconductors to be used as sources and detectors of short wavelength optical radiation, including substrates, thin films, and prototype devices

\section{Constituency:}

Information technology and communications industries that require short-wavelength lasers for high-capacity optical data storage; manufacturing industries that require green, blue, and violet light-emitting devices for full-color solid-state displays such as notebook computers, automotive and aircraft instrumentation panels

Major current tasks:

- Characteristics of intrinsic and defect electronic states by luminescence spectroscopy (high-spatial-resolution CL, PL emission and excitation, time-resolved PL, low-temperature measurements)

- Structure and composition by Raman spectroscopy

- Optical constants by spectrophotometry

- Materials studied include group III nitrides (AlGaN), ZnSe, and other II-VIs

\section{Additional information:}

This program currently involves: (1) a collaboration with Johns Hopkins Applied Physics Laboratory to characterize GaN-AlGaN films and heterostructures being developed for solar-blind UV photodetectors, UV lasers, and blue LEDs; (2) a collaboration with an ATP awardee to investigate ZnSe single-crystal wafers intended for use as substrates for II-VI blue/green LEDs. Major accomplishments include development of facilities for CL and PL spectroscopy of wide-bandgap semiconductors; identification of electronic states associated with dislocations and other defects in $\mathrm{ZnSe}$ wafers by low-temperature CL spectroscopy; imaging of defect structures in thin AlGaN films by spectrally resolved CL, secondary-electron, and optical microscopies. 


\section{Ferroelectric Oxide Thin Films for Photonics}

Project Leader/Contact:

Debra L. Kaiser, (301) 975-6759, dkaiser@nist.gov

Approximate staff (FTE):

2.3 Professionals

Funding sources:

NIST $(100 \%)$

\section{Objective:}

Address key issues in ferroelectric oxide thin films for photonics through a detailed study of the processing/structure/property relationships and the development of measurement techniques for thin films.

\section{Constituency:}

Information technology and communications industries interested in utilizing ferroelectric oxide based devices (electro-optic modulators and switches, optical memory and processing devices, frequency doublers)

Major current tasks:

- MOCVD of epitaxial $\mathrm{BaTiO}_{3}$ thin films

- Characterization of the film composition and microstructure (XRD, SEM, TEM, HREM, AFM, SIMS, WDS, Raman spectroscopy)

- Optical constants by spectrophotometry and prism coupling

- Second harmonic generation (SHG) measurements

- Electrical poling monitored in situ by SHG and polarimetry

- Waveguiding losses by prism coupling

\section{Additional information:}

This program was begun in FY 92 as a NIST Competence Building Initiative. Major accomplishments to date are as follows: (1) designed and constructed a specialized MOCVD system for depositing oxide films; (2) deposited epitaxial $\mathrm{BaTiO}_{3}$ films on $\mathrm{MgO}$ substrates at record low temperature of $600^{\circ} \mathrm{C}$; and (3) established a facility for measuring second harmonic generation, electro-optic coefficients and photorefractive properties. NIST staff members have collaborated with three companies who are fabricating epitaxial ferroelectric thin films by MOCVD for use in photonic devices. The staff members have characterized films from these companies by TEM, Raman spectroscopy, and SHG measurements to aid in the optimization of the thin film deposition processes and the development of processing/structure/property relationships. 


\section{Processing and Lifetime of Light Emitting Polymers}

\section{Project Leader/Contacts:}

G. Thomas Davis (301)975-6725, gtdavis@nist.gov

Emile E. Ettedgui (301)975-4342, emile.ettedgui@nist.gov

Approximate staff (FTE):

1.5 Professionals, 0.5 Contractor/Student

\section{Funding sources:}

NIST $(100 \%)$

\section{Objective:}

Determine the important parameters and underlying mechanisms responsible for the time-dependent decay of light intensity from electroluminescent polymer devices.

\section{Constituency:}

Developers of advanced electronic materials; manufacturers of flat panel displays (computers, TVs, instrument panels, etc.)

\section{Major current tasks:}

- Develop fundamental insights on processing of defect-free thin films of electroluminescent polymer

- Isolate environmental factors that influence electroluminescent lifetime

- Correlate changes in polymer or electrodes with electroluminescent performance

\section{Additional information:}

Research quantities of many different light-emitting (LE) polymers exist. These materials emit light upon the application of an electric field across thin films (ca. $100 \mathrm{~nm}$ ) of the material. The wavelength of emitted light depends upon the composition of the polymer and all primary colors have been demonstrated. A major obstacle to the commercial exploitation of nearly all LE polymers is the decay in light intensity with time of application of the voltage. NIST scientists are collaborating with researchers at the University of Massachusetts in an effort to understand the mechanisms responsible for this limited lifetime in order to guide solutions to the problem. 
Materials Science and Engineering Laboratory

Metallurgy Division

Magnetic Materials Group, 855.11

\section{Magneto-Optical Indicator Film (MOIF) Technique}

\section{Project Leader/Contacts:}

Robert Shull (301)975-6035, robert.shull@nist.gov

Alexander Shapiro (301)975-5970, shapiro@enh.nist.gov

Approximate staff (FTE):

0.5 Professional

Funding sources:

NIST (100\%)

Objective:

To develop a technique for real-time observation and evaluation of local magnetic flux penetration in magnetic domain structure and its dyamic as the external magnetic field and temperature are varied.

\section{Constituency:}

High- $T_{c}$ superconductor manufactures. magnetic recording industry

\section{Major current tasks:}

- Observation of domain kinetics in G.MR devices

- Determination of flux trapping sites in high $T_{c}$ materials

- Observation of domain structure in magnetic nanocomposites

\section{Additional information:}

Magneto-optical indicator film (MOIF) technique uses the Bi-substituted yttrium iron garnet film with in-plane anisotropy, placed on a specimen to be studied, for observation of the magnetic stray fields above the specimen through the magneto-optical Faraday effect created in the garnet film. The resulting optical image of the stray magnetic fields is observed in a polarized light optical microscopy. This technique is much more simple and no less sensutwe than well known methods such as magneto-optical Kerr effect, magnetic force microscopy, or scanning electron microscopy with polarization analysis. In addition, the MOIF technique provides information about bulk domain structure and its dynamics.

Materials currently under study are magnetic multilayer and spin-valve structures, granular magnetic thin films, and nanostructured materials. 


\section{Hybrid-Optical Pattern Recognition and Neural Networks}

\section{Project Leader/Contacts:}

Charles Wilson, (301)975-2080, cwilson@nist.gov

Eung-Gi Paek, (301)975-2195, paek@nist.gov :

Approximate staff (FTE):

3 Professionals

\section{Funding sources:}

NIST (80\%), Other Agency (20\%)

\section{Objective:}

To develop a hybrid optical pattern recognition system that is capable of recognizing patterns, characters and faces. Also, develop measurement methods to increase accuracy of the optical system.

\section{Constituency:}

Federal Bureau of Investigation

\section{Major current tasks:}

- Implementation of hybrid optical signal processing and pattern recognition

- Comparison with digital computer simulation results

- Explore measurement issues to increase accuracy of the system

- Develop test and measurement methods

\section{Additional information:}

Optical pattern recognition and signal processing have long been one of the major application areas of optoelectronic devices. One of the main hurdles for the practical realization of a photonic information processing systems is the lack of accuracy and reliability. Our research is focused on the improvement of accuracy of an optical system by developing correct measurement methods. Also, we will explore a variety of applications of hybrid optical information processing for commercial use such as optical security. 


\section{Volume Holographic Storage}

\section{Project Leader/Contacst:}

Charles Wilson, (301)975-2080, cwilson@nist.gov

Eung-Gi Paek, (301) 975-2195, paek@nist.gov

Approximate staff (FTE):

3 Professionals

\section{Funding sources.}

NIST (80\%), Other Agency (20\%)

\section{Objective:}

To develop a volume holographic storage system with large storage capacity and fast access. Main focus is to develop test/measurement/evaluation methods at the component and system level.

\section{Constituency:}

Federal Bureau of Investigation

\section{Major current tasks:}

- Characterization of photorefractive crystals

- Test/evaluation methods of spatial light modulators (SLMs) and CCDs

- Nondestructive readout/fixing

- Exposure schedule for multiple holograms

- Efficient multiplexing scheme (wavelength, angle, space, fractal, electric, phase, etc.)

\section{Additional information:}

Recent multimedia applications, including video-on-demand, have increased the demand for storage devices with large capacity and fast access. Conventional storage devices that include slow mechanical moving parts may soon find limitations in satisfying the speed requirements of multimedia applications. Recent resurgence of volume holographic storage is mainly motivated by new optoelectronic devices such as spatial light modulators, lasers, detectors, modulators, and deflectors. This project is focused on the development of new measurement methods to test, evaluate, and characterize the holographic storage at both the system and component level. 


